\title{
Avaliação biomecânica de diferentes métodos de fixação tibial na reconstrução do ligamento anterolateral em ossos suínos*
}

\section{Biomechanical Evaluation of Different Tibial Fixation Methods in the Reconstruction of the Anterolateral Ligament in Swine Bones}

\author{
Rogério Nascimento Costa ${ }^{1}$ Rubens Rosso Nadal ${ }^{1}$ Paulo Renato Fernandes Saggin ${ }^{1}$ \\ Osmar Valadão Lopes Junior ${ }^{1}$ Leandro de Freitas Spinelli ${ }^{2}$ Charles Leonardo Israel ${ }^{2}$
}

1 Serviço de Cirurgia do Joelho, Hospital São Vicente de Paulo, Passo Fundo, RS, Brasil

2 Laboratório de Bioengenharia, Biomecânica e Biomateriais, Universidade de Passo Fundo, Passo Fundo, RS, Brasil

\author{
Address for correspondence Rogério Nascimento Costa, Serviço de \\ Cirurgia do Joelho, Hospital São Vicente de Paulo, Passo Fundo, RS, \\ Brasil (e-mail: rogerioortopediasantacasa@hotmail.com; \\ pesquisa2@hsvp.com.br).
}

Rev Bras Ortop 2019;54:183-189.

\section{Resumo \\ Palavras-Chave \\ - ligamento cruzado anterior \\ - ligamentos articulares \\ - joelho \\ - procedimentos ortopédicos}

Objetivo Avaliar a força de resistência à tração de diferentes métodos de fixação tibial na reconstrução do ligamento anterolateral (LAL). Além disso, comparar os mecanismos de falha da fixação tibial dessa reconstrução em joelhos suínos.

Métodos Foram usados 40 membros recém-congelados de suínos, divididos em quatro grupos de dez espécimes, conforme as técnicas de fixação tibial usadas. No grupo A, a fixação tibial do enxerto tendíneo foi feita por meio de uma âncora e seu fio transpassou o enxerto. No grupo B, a fixação tibial foi feita por meio de parafuso de interferência metálico em túnel ósseo único. No grupo C, a fixação tibial incluiu uma âncora associada à sutura de ponto sobre o tendão (sem a presença de fio que transpassasse o tendão) e, no grupo $D$, foram usados dois túneis ósseos confluentes associados a um parafuso de interferência em um dos túneis.

Resultados A força média menos elevada $(70,56 \mathrm{~N})$ ocorreu no grupo $\mathrm{A}$ e a mais elevada $(244,85 \mathrm{~N})$, no grupo $\mathrm{B}$; as médias dos outros dois grupos variaram entre $171,68 \mathrm{~N}$ (grupo C) e 149,43 N (Grupo D). Considerando-se a margem de erro fixada (5\%), foi observada diferença significativa entre os grupos $(p<0,001)$.

Conclusão A fixação com parafuso de interferência em túnel ósseo único apresentou a maior força de resistência à tração dentre as técnicas avaliadas.

Objective The present study aims to evaluate different methods of tibial fixation in the reconstruction of the anterolateral ligament (ALL). In addition, the present paper aims to compare the effectiveness of these methods and their mechanisms of failure in swine knees.
Trabalho feito no Instituto de Ortopedia e Traumatologia de Passo Fundo, RS, Brasil. Publicado originalmente por Elsevier Ltda. received

July 6,2017

accepted

September 21, 2017
DOI https://doi.org/

10.1016/j.rbo.2017.09.001. ISSN 0102-3616.
Copyright $\odot 2019$ by Sociedade Brasileira License terms de Ortopedia e Traumatologia. Published by Thieme Revnter Publicações Ltda, Rio de Janeiro, Brazil 


\author{
Keywords \\ - anterior cruciate \\ ligament \\ - articular ligaments \\ - knee \\ - orthopedic \\ procedures
}

\begin{abstract}
Methods A total of 40 freshly frozen swine limbs were divided into 4 groups of 10 specimens, according to the tibial fixation technique used. In group A, the tibial fixation of the tendon graft was made through an anchor passing the graft. In group $B$, the tibial fixation was performed through a metal interference screw in a single bone tunnel. In group C, the tibial fixation included an anchor associated with a tendinous suture (but not with a wire crossing the tendon). In group $D$, two confluent bony tunnels were drilled and combined with an interference screw in one of them.

Results The lowest mean force $(70.56 \mathrm{~N})$ was observed in group A, and the highest mean force $(244.85 \mathrm{~N})$ was observed in group $\mathrm{B}$; the mean values in the other 2 groups ranged from $171.68 \mathrm{~N}$ (group $C$ ) to $149.43 \mathrm{~N}$ (group D). Considering the margin of error $(5 \%)$, there was a significant difference between the groups $(p<0.001)$.

Conclusion Fixation with an interference screw in a single tunnel bone showed the highest tensile strength among the evaluated techniques.
\end{abstract}

\section{Introdução}

Rupturas do ligamento cruzado anterior (LCA) estão entre as lesões mais comuns entre atletas. ${ }^{1,2}$ No entanto, em até $25 \%$ das reconstrução do LCA os pacientes relatam instabilidade rotacional residual, evidencia que a capacidade de restaurar completamente a estabilidade no plano axial com cirurgias para reconstrução isolada do LCA continua a ser um desafio. ${ }^{3}$ Recentemente, o ligamento anterolateral (LAL) foi descrito e reconhecido como um contribuinte potencial para a frouxidão rotatória. ${ }^{2,4,5}$ Essa estrutura foi primeiramente descrita por Segond,6 em 1879, como uma "faixa fibrosa perolada que era evidente em rotação interna"6,7 e ao longo de mais de 130 anos houve várias descrições diferentes do LAL, mas poucas realmente focadas em sua estrutura. A anatomia e as propriedades biomecânicas do ligamento anterolateral foram descritas recentemente, ${ }^{8-10}$ constatou-se sua importância para restaurar a função completa durante a reconstrução do LCA. ${ }^{2}$ Acredita-se que a reconstrução do LAL pode reduzir o pivot-shift residual após uma reconstrução intra-articular e, assim, melhorar a cinemática pós-operatória do joelho. ${ }^{11}$

Diversas técnicas descrevem reconstrução do LAL, entretanto não há um padrão-ouro determinado para fixac,ão desse ligamento.

Na reconstrução do LCA, tem sido observado um índice de falha entre $4 \%^{12}$ e $27,3 \%{ }^{13}$ com autoenxertos isquiotibias. No entanto, essa percentagem pode ser subestimada, uma vez que o número exato de reconstruções do LCA com falha é difícil de calcular. ${ }^{14}$ Analogamente às reconstruções do LCA, é primordial a aplicação de uma técnica de fixação segura para suportar as forças sobre o enxerto nas reconstruções do LAL e permitir protocolos de reabilitação que possibilitem descarga de peso e retorno precoce à atividade esportiva. ${ }^{15}$

Este estudo tem como objetivo analisar a força de tração necessária para falha da inserção tibial do enxerto em quatro métodos de fixação tibial comumente usados na reconstrução do ligamento anterolateral.

\section{Material e Métodos}

Foi analisada biomecanicamente em laboratório a força de tração, em Newtons $(\mathrm{N})$, necessária para a falha de quatro diferentes modos de fixação usados na reconstrução do LAL. Para isso, 40 membros traseiros recém-congelados de suínos foram divididos em quatro grupos de 10 espécimes, conforme o método de fixação tibial usado. No grupo A, a fixação tibial do enxerto tendíneo foi feita através de uma âncora (Hexagon) e seu fio (Ultrabraid da Smith \& Nephew) transpassou o enxerto. No grupo B, a fixação tibial foi feita através de parafuso de interferência metálico (Traumédica $-7 \times 20 \mathrm{~mm}$ ) em túnel ósseo único. No grupo $C$, a fixação tibial incluiu uma âncora (Hexagon) associada à feitura de ponto sobre o tendão (sem a presença de fio que transpassasse o tendão) e, no grupo D, foram usados dois túneis ósseos confluentes associados a parafuso de interferência (Traumédica - $7 \times 20 \mathrm{~mm}$ ) em um dos túneis. Todas as peças anatômicas usadas foram retiradas de animais com aproximadamente $100 \mathrm{Kg}$ de peso vivo e entre oito e nove meses. Nenhuma das amostras apresentava sinais de lesões prévias ou doença degenerativa e todas tinham a articulação do joelho estável funcionalmente. Os joelhos suínos foram dissecados uniformemente. Todas as estruturas periféricas do joelho foram seccionadas e extirpadas, de maneira que permanecesse apenas a tíbia ( - Fig. 1). Os tendões extensores dos dedos do pé dos suínos ${ }^{16}$ foram dissecados e retirados para ser usados como enxerto tendíneo e padronizados com $12 \mathrm{~cm}$ de comprimento e $4 \mathrm{~mm}$ de diâmetro (-Fig. 2). Foi medida a largura de cada platô tibial para padronização das amostras (-Fig. 3 e - Tabela $\mathbf{1}$ ).

\section{Técnica cirúrgica}

Após remoção de todos os músculos, ligamentos e cápsulas articulares dos joelhos suínos, as tíbias foram preparadas para o ensaio. Tendões extensores dos dedos foram dissecados e extraídos dos pés das peças, posteriormente todas as amostras foram armazenadas a -20 C durante 24 a 48 horas, até serem 


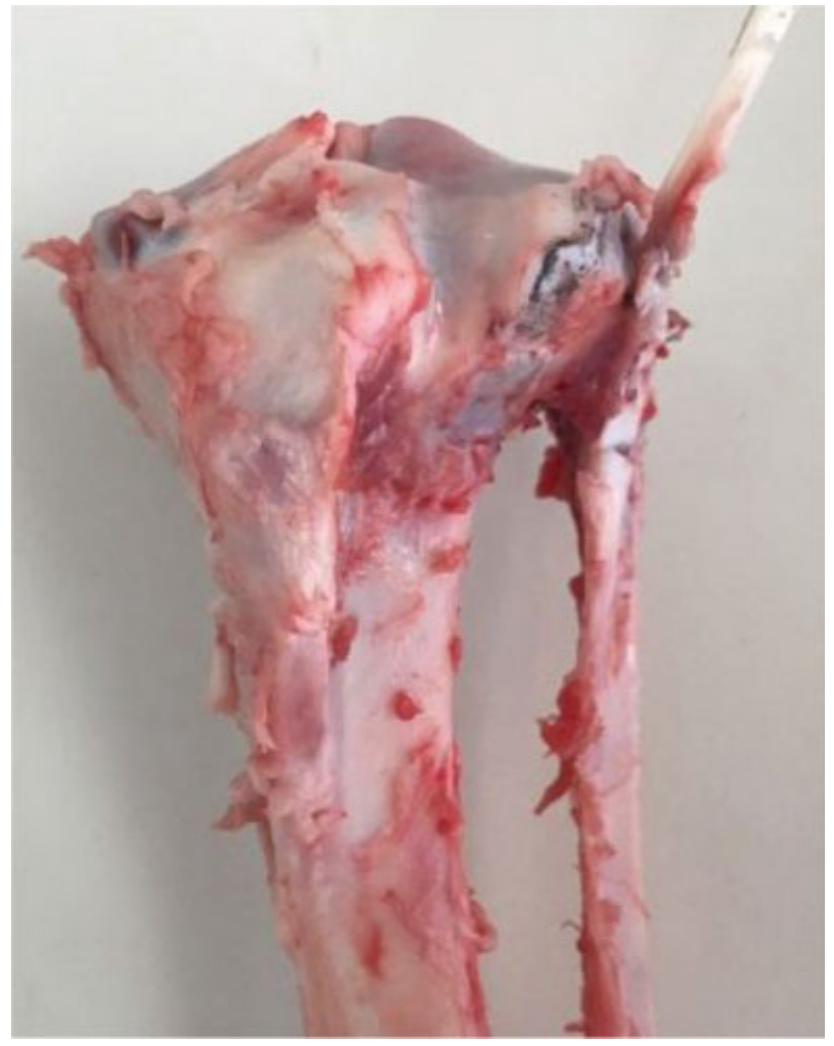

Fig. 1 Preparação da tíbia.

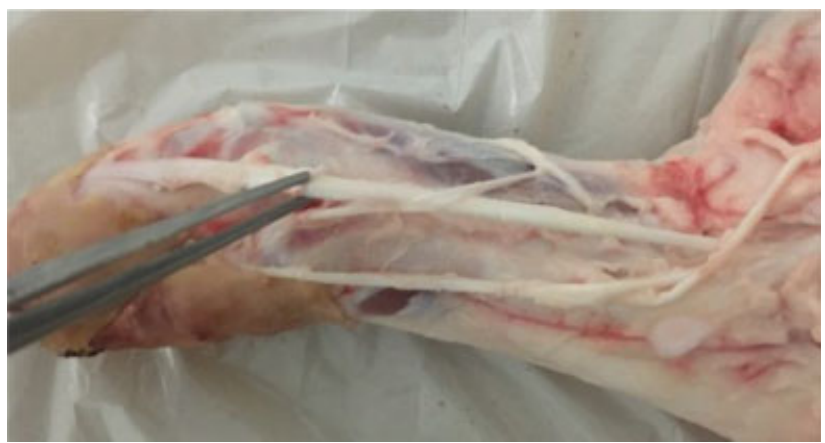

Fig. 2 Preparação do enxerto.

usadas para os testes. Antes do ensaio, cada amostra foi descongelada durante a noite a $4 \circ \mathrm{C}$. Os espécimes foram testados entre 24 e 72 horas após o descongelamento. Todos os testes foram feitos à temperatura ambiente e as amostras foram constantemente mantidas em recipiente úmido com solução salina. Todos os métodos de fixação foram fixados no local considerado como a inserção tibial anatômica do ligamento anterolateral entre a cabeça da fíbula e tubérculo de Gerdy ${ }^{16}$ conforme descrito por Vicente et al ${ }^{17}$ e Helito et al. ${ }^{5,18}$

\section{Preparação das amostras}

Após o preparo das amostras, foi feita a fixação do enxerto na tíbia, em cada grupo, conforme o método.

\section{Grupo A}

Enxerto tendíneo fixado com uma âncora (Hexagon) de $5 \mathrm{~mm}$ e sutura (fio Ultrabraid da Smith \& Nephew) que

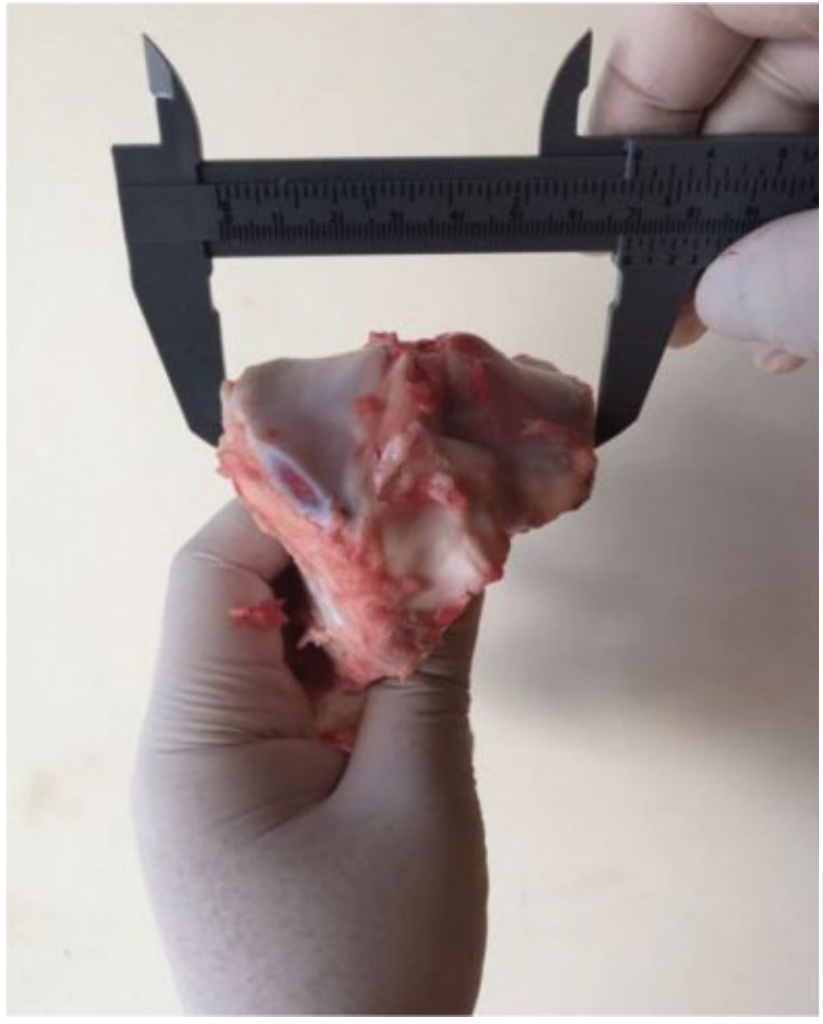

Fig. 3 Largura do platô.

Tabela 1 Medida da distância mediolateral do platô tibial (mm)

\begin{tabular}{|l|l|l|l|}
\hline Grupo & Média & Desvio-padrão & $\begin{array}{l}\text { Coeficiente de } \\
\text { variação (\%) }\end{array}$ \\
\hline A & 66,70 & 2,41 & 3,61 \\
\hline B & 65,90 & 2,85 & 4,32 \\
\hline C & 66,90 & 3,84 & 5,74 \\
\hline D & 67,90 & 2,38 & 3,51 \\
\hline
\end{tabular}

transpassou pelo tendão, foram feitos cinco nós de cirurgião ${ }^{19}$ (Técnica de Pauchet - Fig. 4A).

\section{Grupo B}

Enxerto tendíneofixado através de parafuso de interferência metálico (traumédica - $7 \times 20 \mathrm{~mm}$ ) dentro de túnel ósseo de $6 \times 30 \mathrm{~mm}$ feito a meia distância entre a cabeça da fíbula e o tubérculo de Gerdy (-Fig. 4B).

\section{Grupo C}

Enxerto tendíneo fixado com âncora de $5 \mathrm{~mm}$ com cinco nós de cirurgião ${ }^{19}$-Técnica de Pauchet (fio Ultrabraid da Smith \& Nephew) - e sutura sobre o tendão dobrado (a cavaleiro). De maneira que se obtiveram dois "braços", um de $8 \mathrm{~cm}$ (posterior) fixado na célula de carga e o segundo braço de $4 \mathrm{~cm}$ (anterior) no qual foi feita uma sutura proximal e novamente fixação à célula de carga, formou-se assim um enxerto duplo conforme descrito por Bertrand20 (-Fig. 4C). 
A

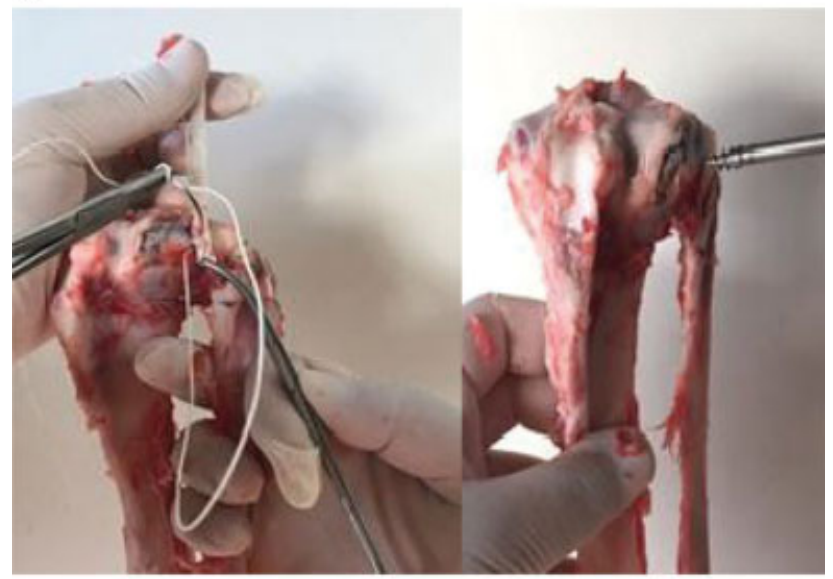

B

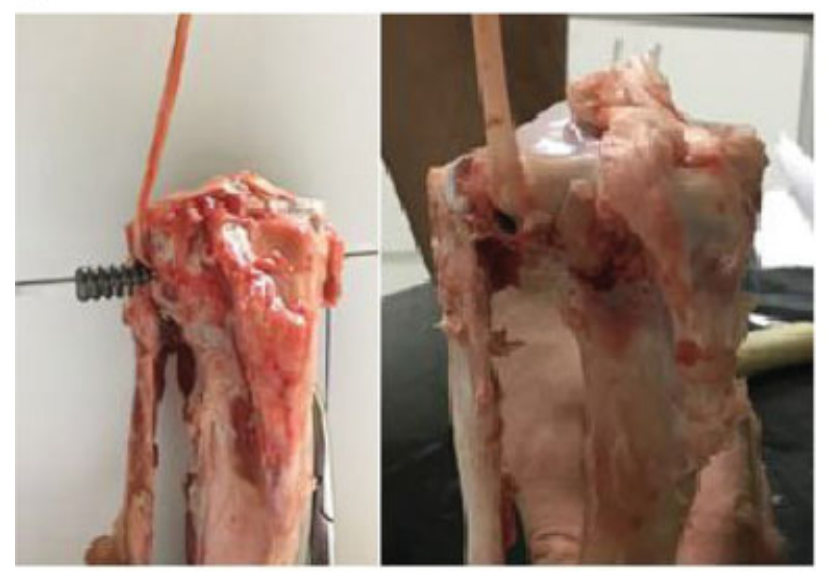

C

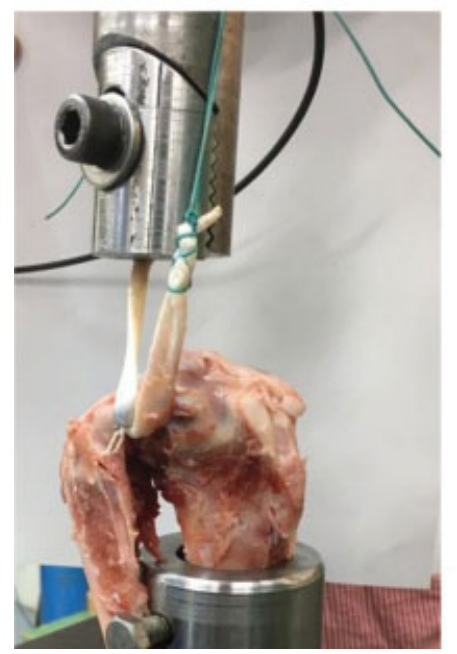

D

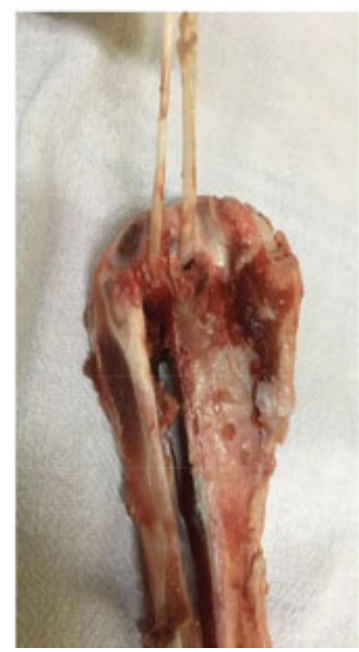

Fig. 4 A, Grupo A. B, Grupo B. C, Grupo C. D, Grupo D.

\section{Grupo D}

Foram confeccionados dois túneis ósseos com broca de 6 $\times 30 \mathrm{~mm}$ em angulações de 90 。 entre si, para comunicação entre os túneis. Feita a passagem do tendão pelos túneis, de modo que o enxerto se apresentasse com dois "braços". O braço do enxerto no túnel posterior com $8 \mathrm{~cm}$ foi fixado com um parafuso de interferência metálico $7 \times 20 \mathrm{~mm}$ e no segundo braço anterior com $4 \mathrm{~cm}$ foi feita uma sutura proximal e fixada também à célula de carga, formou-se assim um enxerto duplo conforme descrito por Bertrand ${ }^{20}$ (- Fig. 4D).

\section{Testes biomecânicos}

Os grupos foram submetidos a um teste biomecânico de tração com o seguinte modelo: Máquina Universal de Ensaio de Tração (Marca: Schenck/Modelo: Upm 200/Cap. 200N 20T, Passo Fundo, RS, Brasil), com uma célula de carga modelo 9 USB da HBM (HBM, Inc. Marlborough, MA, USA) de $20 \mathrm{kgf}$, com uma velocidade de $60 \mathrm{~mm} / \mathrm{min}$. Os parâmetros de força foram registrados no computador através de um sistema de software Spider 8 (HBM, Inc. Marlborough, MA, USA)que é complementado com amplificador para aquisição de dados Catman Easy 3.1 (HBM, Inc. Marlborough, MA, USA), usaramse as rotinas de aquisição fornecidas pelo fabricante do sistema.

As tíbias foram posicionadas abaixo da célula de carga que é fixada à máquina por um suporte tubular preso por um parafuso através do osso ( - Fig. 5 ). 0 tendão foi fixado como enxerto na região proximal da tíbia conforme descrito nos grupos acima, de maneira que cerca de $4 \mathrm{~cm}$ de tendão permaneceram livres e $4 \mathrm{~cm}$ presos na célula de carga, manteve-se o eixo tibial visualmente em alinhamento com o eixo da máquina. Na montagem, o suporte tubular foi fixado na base da máquina com um torno de bancada, permite apenas o deslocamento proximal do tendão, que foi anexado ao elemento móvel da máquina. $O$ teste foi feito com a tíbia em aproximadamente 30。 de flexão, simulou o ângulo de tração do LAL.

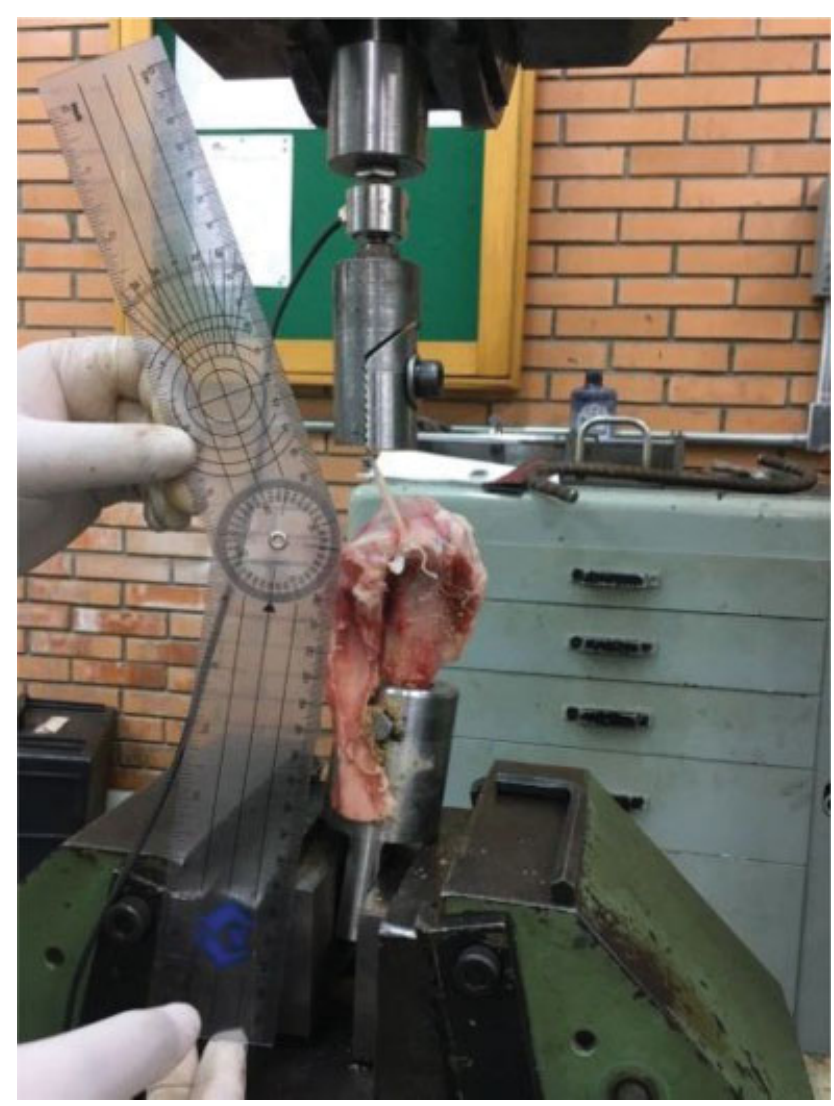

Fig. 5 Posição da tíbia durante os testes. 
Os parâmetros avaliados foram: a força no limite de resistência máxima dos diversos tipos de fixação do ligamento, expressa em Newtons; e os modos de falhas. Foi definido como falência (falha) da amostra quando a curva do gráfico $(\mathrm{N})$ apresentava uma queda súbita durante o teste. $\mathrm{O}$ teste então era interrompido e o gráfico analisado preliminarmente.

Este estudo foi aprovado pela Comissão de Ética no uso de animais da nossa Universidade sob o número CEUA 039/ 2016.

\section{Análise estatística}

Os dados foram expressos através de média, desvio-padrão e coeficiente de variação para as variáveis numéricas e frequências absolutas para a variável categórica.

O tamanho da amostra foi determinado com dados de estudos anteriores. ${ }^{9,21,22}$ Para a comparação entre os grupos em relação à força de tração foi usado o teste $\mathrm{F}$ (Anova) com comparações múltiplas de tamanho. A verificação das hipóteses de normalidade dos dados e de igualdade de variâncias foi feita, respectivamente, através dos testes de Shapiro-Wilk e Levene e o uso das comparações de tamanho foi devido à rejeição da igualdade de variâncias entre os grupos. ${ }^{22,23}$

A margem de erro usada na decisão dos testes estatísticos foi de 5,0\%. Os dados foram digitados na planilha Excel e o programa usado para obtenção dos cálculos estatísticos foi o SPSS versão $23 .{ }^{22,23}$

\section{Resultados}

$\mathrm{Na} \boldsymbol{- T a b e l a} 1$ são apresentadas as estatísticas da largura do platô segundo o grupo, no qual se destaca uma variabilidade bastante reduzida.

Os coeficientes de variação foram, no máximo, iguais a $5,74 \%$, evidenciaram uma homogeneidade elevada em cada grupo em relação ao tamanho das tíbias.

$\mathrm{Na}$ - Tabela 2 apresentam-se as médias da força, segundo o grupo. Para a margem de erro fixada (5\%) se comprova diferença significativa entre os grupos $(\mathrm{p}<0,001)$ e através dos testes de comparações múltiplas (entre os pares de grupos) verifica-se que, com exceção dos grupos C e D, nos demais pares de grupos existem diferenças significativas.

Tabela 2 Média da força segundo o grupo

\begin{tabular}{|l|l|l|l|}
\hline Grupo & Média & Desvio-padrão & $\begin{array}{l}\text { Coeficiente de } \\
\text { variação (\%) }\end{array}$ \\
\hline $\mathrm{A}$ & $70,56^{\mathrm{a}}$ & 12,49 & 17,70 \\
\hline $\mathrm{B}$ & $244,85^{\mathrm{b}}$ & 34,89 & 14,25 \\
\hline $\mathrm{C}$ & $171,68^{\mathrm{C}}$ & 21,56 & 12,56 \\
\hline $\mathrm{D}$ & $149,43^{\mathrm{C}}$ & 85,44 & 57,17 \\
\hline \multicolumn{4}{|l}{ Valor de $\mathrm{p}^{\mathrm{a}}<0,001^{*}$} \\
\hline
\end{tabular}

*Diferença significativa a 5\%. ${ }^{a}$ Através do teste $\mathrm{F}$ (Anova) com comparações através do teste de tamanho. Se as letras subscritas são distintas se comprova diferença significativa entre os grupos correspondentes.
Tabela 3 Tipo de falhas segundo o grupo

\begin{tabular}{|l|l|l|l|l|}
\hline \multirow{2}{*}{ Tipos de falha } & \multicolumn{4}{|l|}{ Grupo } \\
\cline { 2 - 5 } & $\begin{array}{l}\text { A } \\
(\boldsymbol{n})\end{array}$ & $\begin{array}{l}\text { B } \\
(\boldsymbol{n})\end{array}$ & $\begin{array}{l}\text { C } \\
(\boldsymbol{n})\end{array}$ & $\begin{array}{l}\text { D } \\
(\boldsymbol{n})\end{array}$ \\
\hline Ruptura do enxerto & 9 & 2 & - & 3 \\
\hline Soltura do nó & 1 & - & - & - \\
\hline $\begin{array}{l}\text { Deslizamentos do enxerto } \\
\text { do túnel tibial }\end{array}$ & - & 8 & - & - \\
\hline $\begin{array}{l}\text { Soltura do nó proximal } \\
\text { do segundo braço }\end{array}$ & - & - & 7 & - \\
\hline Soltura do nó distal & - & - & 2 & - \\
\hline Soltura da âncora & - & - & 1 & - \\
\hline Quebra da cortical & - & - & - & 6 \\
\hline Soltura fixação femoral & - & - & - & 1 \\
\hline Total & 10 & 10 & 10 & 10 \\
\hline
\end{tabular}

A variabilidade expressa através do coeficiente de variação se mostra razoavelmente elevada no grupo D, no qual o valor da referida medida foi superior a $50 \%$, e pode ser considerada reduzida nos demais grupos em que a medida foi inferior a $33,3 \%$.

$\mathrm{Na}$ - Tabela 3 apresentam-se os tipos de falhas segundo o grupo. Destacam-se as mais frequentes: nove casos de falha por ruptura do enxerto no grupo 1; oito casos de deslizamento do enxerto do túnel tibial no grupo 2; sete casos de soltura do nó proximal do segundo braço no grupo 3 e seis tíbias com quebra de cortical no grupo D.

\section{Discussão}

Os modelos foram submetidos a testes biomecânicos, que permitiram a comparação direta de diferentes técnicas e dispositivos de fixação do enxerto substituto do LAL em tíbias suínas.

Observa-se que a fixação com parafusos de interferência em um único túnel ósseo apresentou a maior resistência à tração para a reconstrução do LAL, estava adequada à necessidade do ligamento nativo no humano apresentado no estudo de Camilo et al $^{24}$ que demonstram a resistência biomecânica do LAL, que tinha uma força máxima de 204,8 $\mathrm{N}$ e uma rigidez média de 41,9 N/mm. Esses resultados permitem a seleção de enxertos adequados e métodos de fixação para possíveis reconstruções do LAL associados com reconstruções do LCA. ${ }^{24}$ Isso sugere que as bandas simples de todos os enxertos, autólogo ou homólogo, comumente usados na prática clínica para a reconstrução do ligamento ao redor do joelho têm as características de resistência biomecânicas necessárias para todas as reconstruções ${ }^{24}$ e reforça a importância de métodos de fixação "suficientes".

A fixação de tecidos moles, tais como ligamentos, ao osso é rotineiramente usada na cirurgia ortopédica e na medicina desportiva. ${ }^{25,26}$ A fixação com parafuso de interferência proporciona um encaixe com pressão entre o osso e 0 enxerto/tendão e o parafuso, é frequentemente usada para anexar ligamentos de substituição em túneis perfurados para 
a reconstrução ligamentar. As âncoras de sutura são usadas em procedimentos cirúrgicos em que é necessário que um cirurgião fixe um tecido à superfície óssea. ${ }^{26} \mathrm{O}$ método ideal deve fornecer uma fixação mecânica adequada e com força igual ou superior àquelas ocorridas na reabilitação, antes que ocorra a integração do enxerto tendíneo ao osso. ${ }^{25,26}$

Nos grupos A e C, nos quais foram usadas âncoras como método de fixação, evidenciou-se que o uso de sutura transtendão pode fragilizá-lo e deixá-lo mais vulnerável a cargas de tração mais baixas. Isso é exemplificado pela inferioridade média do grupo A (70N) em comparação com o grupo C (149N). No entanto, as âncoras permitem a inserção direta de implantes (sem necessidade de perfuração, rosqueamento ou pré-perfuração) com o uso de ponta de autoinclusão, bem como aperfeiçoamento e tensionamento de suturas individuais. As âncoras de sutura produzem tensão em toda a interface tendão-osso. O tamanho e o posicionamento do implante em osso cortical ou esponjoso determinam a força de fixação. ${ }^{21}$ Barber et $\mathrm{al}^{27}$ concluíram que algumas âncoras de sutura mais recentes mostraram melhorias significativas nos valores de carga a falha quando comparadas com suturas de poliéster traçado. Logo, observa-se que o tipo de material de sutura também influência no modo de falha. ${ }^{27}$

No presente estudo, os grupos B e D receberam fixação com parafuso de interferência. No grupo B foi feito apenas um túnel ósseo, já no grupo $\mathrm{D}$ foram feitos dois túneis divergentes que se comunicavam para a passagem do enxerto, conforme relatado. O grupo B apresentou maior força de tração em comparação com os outros grupos, ao passo que o grupo $\mathrm{D}$ apresentou falha precoce em todas as amostras, como a quebra da cortical e uma variação da força de tração com um coeficiente de variação razoavelmente elevada. Logo, conclui-se que essa técnica cirúrgica não é reprodutível em osso suíno, são então necessários outros testes em ossos humanos. Na análise dos padrões de falha (-Tabela 3 ) resultantes da resistência à tração em cada grupo, mostrou-se que a falha se dava de forma variada, dependia do método de fixação, e que prevalecia um tipo de falha em cada grupo.

Um potencial viés de nosso estudo foi o uso de peças de joelhos suínos, Nurmiet $\mathrm{al}^{28}$ relataram que a densidade óssea trabecular da tíbia de porco foi significativamente maior do que a da tíbia humana, tal como avaliado por varrimento quantitativo na tomografia computadorizada periférica. ${ }^{28,29}$ Apesar disso, as tíbias suínas foram usadas muitas vezes em estudos experimentais semelhantes (sobretudo para reconstruções do LCA) e não foram encontradas diferenças significativas sobre o uso do parafuso de interferência para fixação do enxerto em suínos e humanos. ${ }^{21}$ Em um estudo prévio sobre o uso de fixação de enxerto de parafuso de interferência em osso suíno e humano, as diferenças máximas de resistência à tração foram insignificantes. ${ }^{28}$ Comparações diretas entre espécimes humanos são difíceis porque é difícil de controlar fatores como idade do doador e diferenças de densidade óssea. Por essas razões, optamos por usar osso de porco, o que nos permitiu controlar esses fatores. $^{29-31}$

Outro potencial viés diz respeito à localização do LAL, determinada visualmente pelo cirurgião na construção das peças. Tração foi feita linearmente, e não rotacionalmente, como ocorre nos humanos, e de forma lenta, ao invés de rápida, como ocorre em uma entorse.

Em estudos que avaliaram o ponto de fixação tibial do LAL também foi constante e ligeiramente posterior à metade da distância entre o tubérculo de Gerdy e a cabeça da fíbula. Essa fixação pode ser associada com a fratura de Segond, avulsão óssea da tíbia anterolateral encontrada em cerca de $9 \%$ de pacientes com rupturas do LCA. 5,7,32

Este estudo envolveu o teste biomecânico de tempo zero em condições pós-operatórias imediatas. Portanto, nenhuma comparação histológica foi possível. ${ }^{13} \mathrm{E}$ por fim não foram feitos a medição do deslizamento do enxerto para reduzir o erro nem testes de confiabilidade intra ou interobservador.

\section{Conclusão}

A fixação com parafuso de interferência em túnel ósseo único apresentou a maior força de resistência à tração dentre as técnicas avaliadas. Já os túneis convergentes na tíbia suína não foram reprodutíveis, logo necessitam de outros testes em osso humano para melhor avaliação.

Conflitos de interesse

Os autores declaram não haver conflitos de interesse.

\section{Referências}

1 Mall NA, Chalmers PN, Moric M, Tanaka MJ, Cole BJ, Bach BR Jr, et al. Incidence and trends of anterior cruciate ligament reconstruction in the United States. Am J Sports Med 2014;42(10):2363-2370

2 Schon JM, Moatshe G, Brady AW, Cruz RS, Chahla J, Dornan GJ, et al. Anatomic Anterolateral Ligament Reconstruction of the Knee Leads to Overconstraint at Any Fixation Angle. Am J Sports Med 2016;44(10):2546-2556

3 Helito CP, Bonadio MB, Gobbi RG, da Mota E Albuquerque RF, Pécora JR, Camanho GL, et al. Combined intra- and extra-articular reconstruction of the anterior cruciate ligament: the reconstruction of the knee anterolateral ligament. Arthrosc Tech 2015;4(03):e239-e244

4 Sonnery-Cottet B, Thaunat M, Freychet B, Pupim BH, Murphy CG, Claes $\mathrm{S}$. Outcome of a combined anterior cruciate ligament and anterolateral ligament reconstruction technique with a minimum 2-year follow-up. Am J Sports Med 2015;43(07):1598-1605

5 Helito CP, Demange MK, Bonadio MB, Tírico LE, Gobbi RG, Pécora JR, et al. Anatomy and histology of the knee anterolateral ligament. Orthop J Sports Med 2013;1(07):2325967113513546

6 Segond P. Recherchescliniques et experimentalessur les ép anchementssanguins du genou par entorse. Paris: Aux Bureaux du Progres Medical; 1879

7 Claes S, Vereecke E, Maes M, Victor J, Verdonk P, Bellemans J. Anatomy of the anterolateral ligament of the knee. J Anat 2013; 223(04):321-328

8 Kennedy MI, Claes S, Fuso FA, Williams BT, Goldsmith MT, Turnbull TL, et al. The anterolateral ligament: an anatomic, radiographic, and biomechanical analysis. Am J Sports Med 2015;43 (07):1606-1615

9 Parsons EM, Gee AO, Spiekerman C, Cavanagh PR. The biomechanical function of the anterolateral ligament of the knee. Am J Sports Med 2015;43(03):669-674

10 Spencer L, Burkhart TA, Tran MN, Rezansoff AJ, Deo S, Caterine S, et al. Biomechanical analysis of simulated clinical testing and reconstruction of the anterolateral ligament of the knee. Am J Sports Med 2015;43(09):2189-2197 
11 Dodds AL, Gupte CM, Neyret P, Williams AM, Amis AA. Extraarticular techniques in anterior cruciate ligament reconstruction: a literature review. J Bone Joint Surg Br 2011;93(11):1440-1448

12 Maletis GB, Cameron SL, Tengan JJ, Burchette RJ. A prospective randomized study of anterior cruciate ligament reconstruction: a comparison of patellar tendon and quadruple-strand semitendinosus/gracilis tendons fixed with bioabsorbable interference screws. Am J Sports Med 2007;35(03):384-394

13 Beynnon BD, Johnson RJ, Fleming BC, Kannus P, Kaplan M, Samani $\mathrm{J}$, et al. Anterior cruciate ligament replacement: comparison of bone-patellar tendon-bone grafts with two-strand hamstring grafts. A prospective, randomized study. J Bone Joint Surg Am 200284-A(09):1503-1513

14 Kamath GV, Redfern JC, Greis PE, Burks RT. Revision anterior cruciate ligament reconstruction. Am J Sports Med 2011;39(01): 199-217

15 Harvey A, Thomas NP, Amis AA. Fixation of the graft in reconstruction of the anterior cruciate ligament. J Bone Joint Surg $\mathrm{Br}$ 2005;87(05):593-603

16 Getty R. Sisson/Grossman. Anatomia dos animais domésticos. 5a ed. Rio de Janeiro: Interamericana; 1981

17 Vincent JP, Magnussen RA, Gezmez F, Uguen A, Jacobi M, Weppe F, et al. The anterolateral ligament of the human knee: an anatomic and histologic study. Knee Surg Sports Traumatol Arthrosc 2012; 20(01):147-152

18 Helito CP, Miyahara HS, Bonadio MB, Tirico EL, Gobbi RG, Demange MK, et al. Anatomical study of the anterolateral ligament of the knee. Rev Bras Ortop 2013;48(04):368-373

19 Cirino LMI. Manual de técnica cirúrgica para a graduac,ão. São Paulo: Sarvier; 2006

20 Sonnery-Cottet B, Daggett M, Helito CP, Fayard JM, Thaunat M. Combined anterior cruciate ligament and anterolateral ligament reconstruction. Arthrosc Tech 2016;5(06):e1253-e1259

21 Yang DL, Cheon SH, Oh CW, Kyung HS. A comparison of the fixation strengths provided by different intraosseous tendon lengths during anterior cruciate ligament reconstruction: a biomechanical study in a porcine tibial model. Clin Orthop Surg 2014;6(02):173-179
22 Altman DG. Practical statistics for medical research. London: Chapman \& Hall Publishers; 1991

23 Conover WJ. Practical nonparametric statistics. New York: John Wiley \& Sons; 1980

24 Helito CP, Bonadio MB, Rozas JS, Wey JM, Pereira CA, Cardoso $\mathrm{TP}$, et al. Biomechanical study of strength and stiffness of the knee anterolateral ligament. BMC Musculoskelet Disord 2016; 17:193

25 Cole BJ, Sayegh ET, Yanke AB, Chalmers PN, Frank RM. Fixation of soft tissue to bone: techniques and fundamentals. J Am Acad Orthop Surg 2016;24(02):83-95

26 Suchenski M, McCarthy MB, Chowaniec D, Hansen D, McKinnon W, Apostolakos J, et al. Material properties and composition of soft-tissue fixation. Arthroscopy 2010;26(06):821-831

27 Barber FA, Herbert MA, Coons DA, Boothby MH. Sutures and suture anchors-update 2006. Arthroscopy 2006;22;10. 1063.e1$-1069 . e 1$

28 Nurmi JT, Sievänen H, Kannus P, Järvinen M, Järvinen TL. Porcine tibia is a poor substitute for human cadaver tibia for evaluating interference screw fixation. Am J Sports Med 2004;32(03): 765-771

29 Kim MK, Na SI, Lee JM, Park JY. Comparison of bioabsorbable suture anchor fixation on the tibial side for anterior cruciate ligament reconstruction using free soft tissue graft: experimental laboratory study on porcine bone. Yonsei Med J 2014;55(03): 760-765

30 Scheffler SU, Südkamp NP, Göckenjan A, Hoffmann RF, Weiler A. Biomechanical comparison of hamstring and patellar tendon graft anterior cruciate ligament reconstruction techniques: The impact of fixation level and fixation method under cyclic loading. Arthroscopy 2002;18(03):304-315

31 Suggs J, Wang C, Li G. The effect of graft stiffness on knee joint biomechanics after ACL reconstruction-a 3D computational simulation. Clin Biomech (Bristol, Avon) 2003;18(01):35-43

32 Hess T, Rupp S, Hopf T, Gleitz M, Liebler J. Lateral tibial avulsion fractures and disruptions to the anterior cruciate ligament. A clinical study of their incidence and correlation. Clin Orthop Relat Res 1994;(303):193-197 